\title{
Paraplegia
}

\section{Spinal Cord Injury Care System: Fifteen- year Experience at the Rehabilitation Institute of Chicago}

\author{
G.M. Yarkony, MD, ${ }^{1,2,3}$ E.J. Roth, MD, ${ }^{1,2,3}$ P.R. Meyer, Jr, MD, ${ }^{1,2,4}$ L. Lovell, \\ BS, ${ }^{2}$ A.W. Heinemann, PhD, ${ }^{1,2,3}$ H.B. Betts, MD $^{1,2,3}$ \\ ${ }^{1}$ Rehabilitation Institute of Chicago, ${ }^{2}$ Midwest Regional Spinal Cord Injury Care \\ System, ${ }^{3}$ Department of Rehabilitation Medicine, Northwestern University Medical \\ School, ${ }^{4}$ Department of Orthopaedic Surgery, Northwestern University Medical School, \\ Chicago, USA.
}

\section{Summary}

A statistical study of 15 years of the spinal cord injury care system of the Rehabilitation Institute of Chicago is reported. The Rehabilitation Institute of Chicago (RIC) is the rehabilitation component of the Midwest Regional Spinal Cord Injury Care System, a collaborative programme with Northwestern Memorial Hospital and Northwestern University. Data are reported on 1382 patients, a representative sample of the over 2000 patients treated since the inception of the centre. The sample was predominately male $(83 \%, N=1147)$ and caucasian $(64 \%, N=888)$. The most common aetiology was motor vehicle accidents $(36 \%, N=505)$. During the 15-year period there were significant decreases in both acute and rehabilitation lengths of stay. Ninety three per cent of the patients were discharged home. Rehabilitation benefits were demonstrated by improvements in the Modified Barthel Index. The research, educational and clinical programmes are described.

Key words: Spinal cord injury; Rehabilitation Institute of Chicago; Statistical study.

The concept of comprehensive treatment centres for individuals with Spinal Cord Injury (SCI) was instituted in the UK during World War II. Sir Ludwig Guttmann (1976), a neurosurgeon, developed a programme at Stoke Mandeville Hospital which pioneered early concepts of spine management and promoted the concept of reintegration of spinal cord injured individuals into their communities (Young et al., 1982). These concepts included vocational rehabilitation, sports and recreation and served as a model for others worldwide.

In the late 1960s the Cushing Society advocated the establishment of civilian spinal cord injury centres in the USA (Young et al., 1982). In 1970 the first model system in Phoenix was sponsored by the Rehabilitation Services Administration, Department of Health Education and Welfare; now there are 13 Regional Centres in the USA whose goal is to provide comprehensive spinal cord care from injury 
through lifelong follow-up. The purpose of this system of multidisciplinary care is to substantially reduce the loss of life, loss of function, occurrence of serious medical complications and the escalation of spinal cord injury costs. These centres also participate in a co-ordinated system of data collection and research.

In 1972 the Midwest Regional Spinal Cord Injury Care System was established as a combined programme of Northwestern University, Northwestern Memorial Hospital and the Rehabilitation Institute of Chicago (RIC). This programme has been continually federally sponsored. The acute care phase of the programme is housed in a designated 20-bed acute care unit at Northwestern Memorial Hospital and the rehabilitation component is housed at the RIC.

This paper reports the rehabilitation experience of this centre during the 15year period from 1972 to 1986 .

\section{Methods}

Data were collected according to the standards of the National Spinal Cord Injury Statistical Center and its predecessor the National Spinal Cord Injury Data Research Center. To be included in the data collection all individuals met the following criteria:

1. Admitted to the programme within 1 year of injury.

2. Continually hospitalised with no more than 21 days out of the hospital.

3. Complete the rehabilitation programme or were discharged with a normal neurological examination.

4. Give informed consent to participate in data collection.

Individuals who died were also included in the data collection. All individuals had spinal cord injuries due to trauma within the neural canal. Injuries limited to root avulsion or peripheral nerve injuries alone without injury within the neural canal were excluded. Not all individuals with spinal cord injuries admitted to the RIC met this criterion.

Quadriplegia was defined as loss or impairment of motor and/or sensory function in the cervical segments of the spinal cord. Paraplegia was defined as loss of motor and/or sensory function in the thoracic, lumbar, or sacral segments. The definition of paraplegia includes conus medullaris injuries and cauda equina lesions. A complete injury was defined as no sensory or motor function below the zone of cord destruction at the site of primary injury. An incomplete lesion was defined as an injury with any motor and/or sensory function below the zone of cord destruction at the site of primary injury. The zone of injury is up to three neurological segments of the point of damage to the spinal cord where there is frequently some preservation of motor or sensory function. These definitions are consistent with those of the American Spinal Injury Association (1982).

The Modified Barthel Index (MBI) was recorded on 711 patients until 1980. This is a 15-point functional outcome scale which has been shown to be a valid means of describing functional abilities and change over time (Granger et al., 1979). The technique used has been described previously (Yarkony et al., 1987). 


\section{Results}

Data were collected on 1382 patients who met the criteria for the study and gave informed consent. Although this does not include all of the over 2000 SCI patients treated during that time period it is a large and representative sample.

The sample was predominately male, $(83 \%, \mathrm{~N}=1147)$ and caucasian, $(64 \%$, $\mathrm{N}=888$ ). Incomplete quadriplegia was the most common type of injury (30\%), followed by complete quadriplegia (27\%), complete paraplegia (27\%) and incomplete paraplegia (16\%).

Road and traffic accidents were the most common aetiology, (36\%, N=505) followed by penetrating wounds, $(22 \%, \mathrm{~N}=299)$, falls $(20 \%, \mathrm{~N}=278)$, sports $(15 \%$, $\mathrm{N}=206)$ and other causes $(7 \%, \mathrm{~N}=96)$.

The mean age of the population was 27.9 years, the mode was 19 and the median age 25 years. Aetiologies varied with age. Younger patients were most commonly involved in motor vehicle accidents while older patients were more likely to be involved in falls. The most common aetiologies for quadriplegic patients were road and traffic accidents $(23 \%, \mathrm{~N}=318)$ and falls $(12 \%, \mathrm{~N}=166)$. The most common aetiologies for paraplegics were penetrating wounds $(16 \%, \mathrm{~N}=221)$, followed by road and traffic accidents $(14 \%, \mathrm{~N}=194)$.

Aetiology also varied by sex with the most common cause of SCI in males being road and traffic accidents, $(35 \%, \mathrm{~N}=401)$ followed by penetrating wounds $(23 \%$, $\mathrm{N}=262$ ). Females were also most commonly injured by road and traffic accidents $(45 \%, \mathrm{~N}=105)$ but falls were the second most common cause $(21 \%, \mathrm{~N}=50)$ (Fig. 1).

Caucasian patients were most commonly injured by road and traffic accidents $(44 \%, \mathrm{~N}=388)$ followed by falls $(21 \%, \mathrm{~N}=182)$, sports $(20 \%, \mathrm{~N}=181)$, other $(8 \%$, $\mathrm{N}=71$ ) and penetrating wounds $(7 \%, \mathrm{~N}=66)$. Non-caucasian patients' most common aetiology was penetrating wounds $(47 \%, \mathrm{~N}=233)$ followed by road and traffic accidents $(24 \%, \mathrm{~N}=117)$, falls $(19 \%, \mathrm{~N}=96)$ other $(5 \%, \mathrm{~N}=25)$, and sports $(5 \%, N=23)$ (Fig. 1).

Length of stay and days hospitalised prior to admission to RIC has decreased considerably over the years. Patients are now being transferred to RIC in an average of 33.4 days while in 1974 patients were transferred to RIC in a mean of $56 \cdot 5$ days (Fig. 2A). Length of stay at RIC now averages $82 \cdot 8$ days for quadriplegics and 54.3 days for paraplegics with an overall average of 68.1 days. As seen in Figure 2B this has been a considerable decrease over the years. Data for 1972 should not be considered significant as there were 2 admissions during that year. Total days hospitalised has decreased as well (Fig. 2C) with a present mean of $95 \cdot 3$ days.

\section{Discharge status}

Ninety three per cent of patients were discharged to a private residence after rehabilitation (Fig. 3).

Functional outcomes have been described for 711 patients of the sample in a previous paper. Patients at all 4 levels of injury improved their functional abilities from admission to discharge (Fig. 4). Mean MBI Scores improved from 13.8 on admission to $46 \cdot 1$ on discharge for quadriplegics and $37 \cdot 7$ on admission to $74 \cdot 4$ on 
MALE

$7.15 \%$

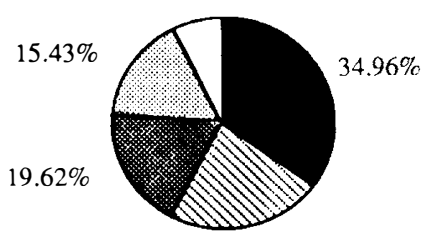

$22.84 \%$

TOTAL $\mathrm{N}=1147$

CAUCASIAN

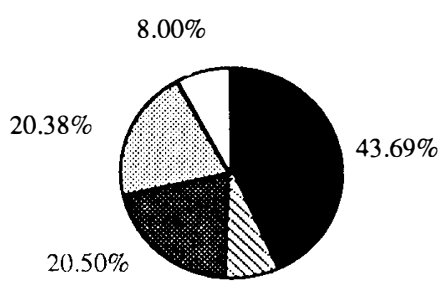

$7.43 \%$

$$
\text { TOTAL } \mathrm{N}=888
$$

FEMALE

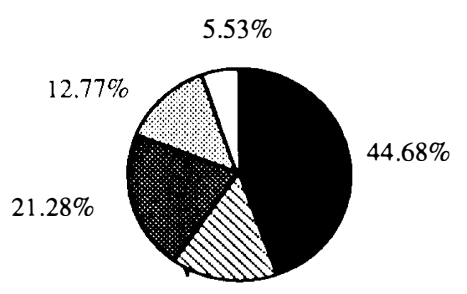

$15.74 \%$

TOTAL $\mathrm{N}=235$

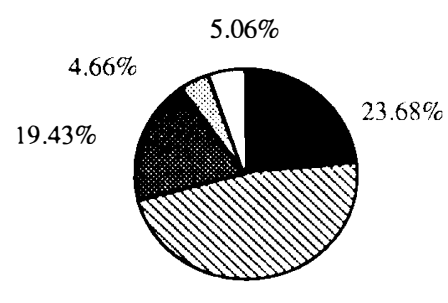

$47.17 \%$

TOTAL N $=494$

\begin{tabular}{|c|}
\hline ROAD TRAFFIC \\
\hline$\otimes$ PENETRATING \\
\hline FALLS \\
\hline SPORTS \\
\hline$\square$ OTHER \\
\hline
\end{tabular}

Figure 1 Aetiology distributed by gender and race.

discharge for paraplegics. Incomplete patients at both levels made greater gains than complete SCI patients. In a 3-year follow-up study of these patients functional skills were maintained or improved (Yarkony et al., 1988). Age did not affect functional abilities in quadriplegic or in incomplete paraplegic patients (Yarkony et al., 1987). Complete paraplegics with advancing age had decreased abilities to 


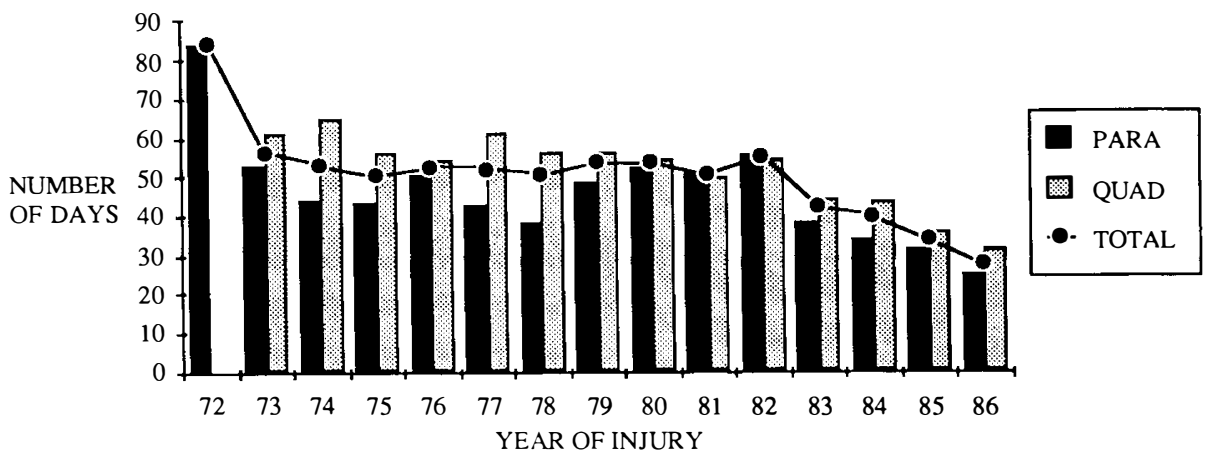

Figure 2A Mean days from injury to rehabilitation admission.

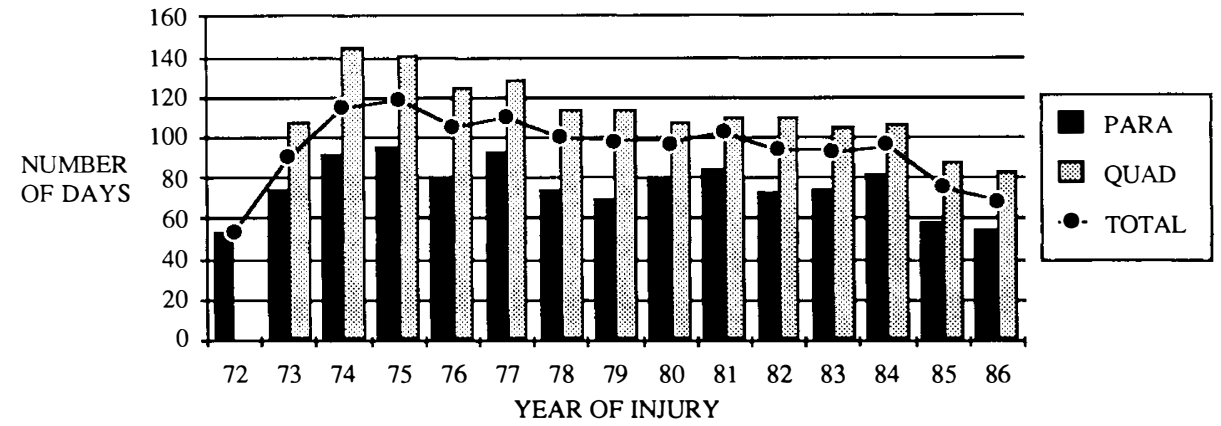

Figure 2B Length of rehabilitation stay by neurological impairment.

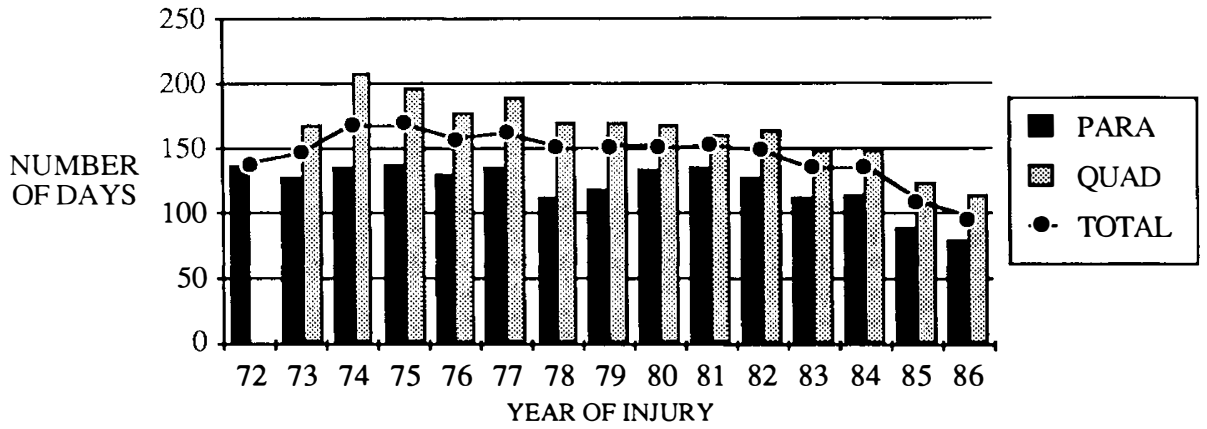

Figure 2C Total combined acute and rehabilitation days for initial hospitalisation.

dress, bathe, perform complex transfers and climb stairs although their total discharge MBI score was not affected by advanced age.

\section{Discussion}

The data shows a decline in the time post-inquiry to admission to the rehabilitation facility. Although we are unable to determine precisely the reason for this decline, 

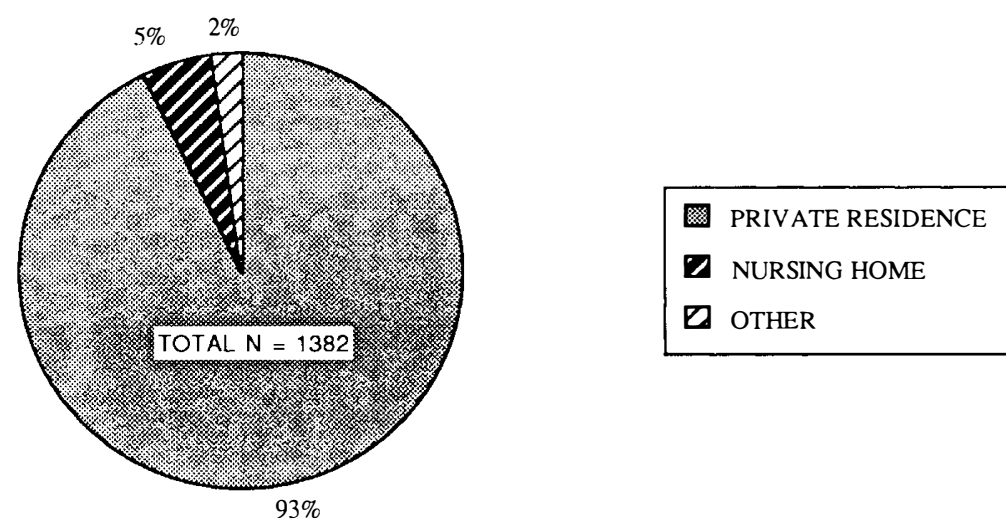

Figure 3 Placement after rehabilitation discharge: 1972-1986.

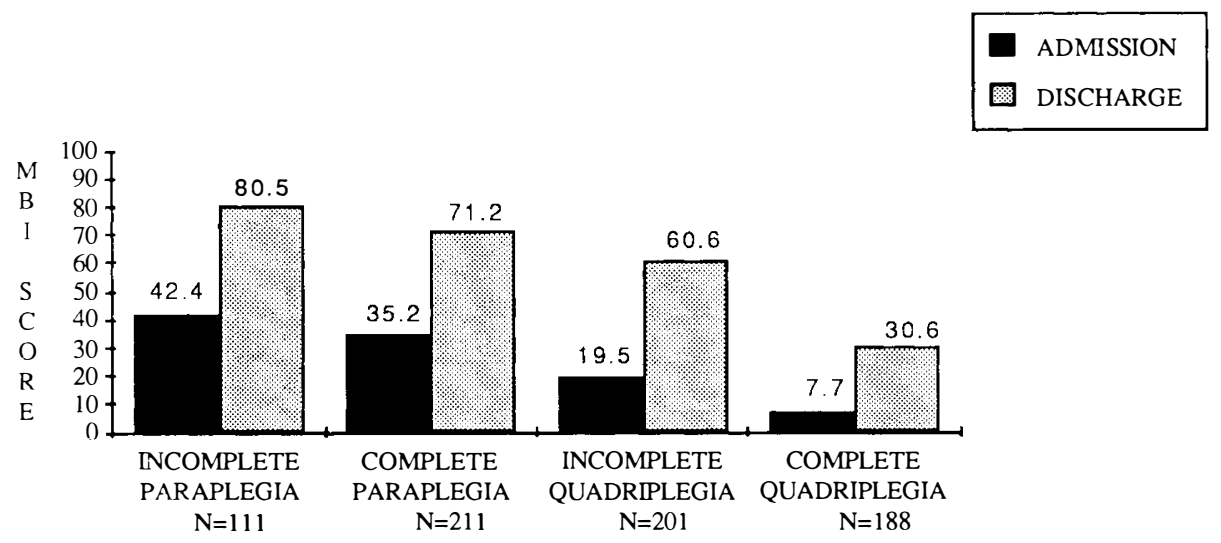

Figure 4 Modified Barthel Index (MBI) scores at admission and discharge. MBI is a 100-point scale used to assess ability to perform self-care and mobility tasks.

we believe it is related to the expertise that develops in a specialised unit with time. This reason applies as well to the declining rehabilitiation length of stay. The relatively high incidence of penetrating wounds seen in our centre is related to the centre's location in a large metropolitan area.

Each of the four patient groups, defined by level and completeness of injury, made functional improvements during rehabilitation hospitalisation as measured by the MBI. Persons with paraplegia were admitted and discharged with greater functional skills. Within the paraplegia and quadriplegia groups, patients with incomplete lesions had greater functional levels.

Functional improvement after spinal cord injury is only part of a total rehabilitiation programme. The objective of rehabilitation is to maximise the patient's recovery from spinal cord injury, and to reintegrate the patient into the community, allowing for competence in independent living and work. When severe disability limits performance in accustomed living patterns, the entire lifestyle and appreciation of life are disrupted. Incapacitation, bewilderment, loss and loneliness affect not only the individual with spinal cord injury but family and friends as well. The likelihood of achieving the goals of rehabilitation is enhanced by an 
integrated, interdisciplinary team of experienced professionals who provide a wide range of services and who develop resources that are needed for a full life (Yarkony et al., 1987). This large series of patients has allowed the development of programmes that have benefited spinal cord injured individuals in our area through patient care, education and research.

The clinical team under the direction of a physician treating spinal cord injured patients at RIC consists of nursing, physical therapy, occupational therapy, clinical psychology, social work, recreation therapy, orthotics, rehabilitation engineering, vocational rehabilitation and chaplaincy. Interdisciplinary collaboration is the key to successful rehabilitation and eventual reintegration of the patient back into his or her community.

The physician co-ordinates the team and manages the medical problems resulting from spinal cord injury. These include management of the neuropathic bowel and bladder, autonomic dysreflexia, heterotopic ossification, respiratory compromise, pressure sores and cardiovascular complications. Programmes to enhance fertility and sexual function in the male with spinal cord injury include vibratory and electroejaculation, and penile injection of vasoactive substances to enhance erections.

Lifelong outpatient follow-up after discharge is co-ordinated by the patient's physician. Nurses work closely with the physician in managing the neuropathic bowel and bladder, and skin problems. Of particular importance is the nurse's role in patient and family teaching. Teaching the management and recognition of such problems as deep venous thrombosis, urinary complications and pressure sores prevent life-threatening complications and decrease the cost of medical care in the future.

Physical therapists teach the patient mobility skills, including bed mobility, transfer skills and gait training with assistive devices such as knee-ankle-foot orthosis and the use of electric powered or manual wheelchairs. Equipment for home use is ordered prior to discharge in collaboration with suppliers in the patient's community.

Occupational therapy deals with the activities of daily living such as feeding, dressing, writing and homemaking. Providing orthotic devices to assist paralysed or weakened muscles to perform these activities is an important part of occupational therapy. High level quadriplegic patients may benefit from the programmes of the Alan J. Brown Center. This centre provides training with sophisticated electronic devices such as environmental controls and computers which can be controlled by mouth sticks or breath control (sip and puff).

Rehabilitation engineering experts collaborate with the therapists in adapting equipment for the individual needs of the patient. Examples of this include special modifications of wheelchairs to provide better posture and prevent pressure sores, improved walker design (Yarkony et al., 1986) and special switching devices for computers, environmental controls and electric wheelchairs.

The clinical psychologist provides support and counselling for the patient and his or her family in adapting to the changes resulting from the injury. Particular areas of importance are sexual functioning and changes in the role of the spinal cord injured individual in his or her family and in the community. An additional role of the psychologist is in neuropsychological evaluation of the patient with concurrent brain injury (Morris et al., 1986). 
Recreational therapy provides diversion for the patient while hospitalised as well as helping him for his future recreational needs. It helps in the resocialisation process by reintroducing the patient to some premorbid interests as well as directing the patient into new areas. A wheelchair sports programme is a major component of this effort.

The goal of vocational rehabilitation is to speed the patients return to work or school. This may involve working with the patient's employer or exploring new interests and career training. A major component of this is driving education which is accomplished by the use of driving simulators followed by behind-the-wheel training.

The social worker serves as a link between the family and the team. Assisting the patient and family in discharge planning is a major role of the social worker. The social worker is active in patient and family counselling as well as in the coordination of educational programmes for patients, families and friends.

Team collaboration is reflected in many of the programmes. The wheelchair seating programme for example is a combined effort of occupational therapy, physical therapy and rehabilitation engineering. The quadriplegic and paraplegic community re-entry programme combines the skills of nursing, recreation and physical and occupational therapy. This collaboration is particularly important in managing quadriplegic patients who are ventilator dependent.

Educational programmes are presented in collaboration with our Education and Training Center. Annual courses are provided on an interdisciplinary basis as well as individual courses for nurses and physical and occupational therapists, psychologists and social workers.

Research efforts are conducted in collaboration with the acute care unit of Northwestern Memorial Hospital. Current programmes at this time include methods to decrease deep venous thrombosis, (Green et al., 1982), enhance fertility, and stand paraplegics with functional neuromuscular stimulation (Yarkony et al., 1987).

\section{References}

American Spinal INJURy Association 1982 Standards for neurological classification of spinal injury patients. American Spinal Injury Association, Chicago.

GRANGER CV, ALBRECHT GL, HAMILTON BB 1979 Outcome of comprehensive medical rehabilitation: measurement by PULSES and the Barthel index. Archives of Physical Medicine and Rehabilitation 60:145-154.

GREen D, Rossi EC, Yoo JST, Fine AR, SPIEs SM 1982 Deep vein thrombosis in spinal cord injury: effect of prophylaxis with calf compression, aspirin and dipyridamole. Paraplegia 20:227234.

GuttmanN L 1976 Spinal Cord Injuries Comprehensive Management and Research, 2nd edn. Blackwell Scientific Publications Inc, Boston.

MORRIS J, ROTH E, DAvidOFF G 1986 Mild closed head injury and cognitive deficits in spinal cordinjured patients: incidence and impact. Foumal of Head Trauma Rehabilitation 1:31-42.

YARKONY GM, JONES R, HEDMANN G, O'DONNELl A 1986 Jones-Hedman walker modification of C7 quadriplegia: case study in team cooperation. Archives of Physical Medicine and Rehabilitiation 67:54-55.

YARKONY GM, ROTH EJ, HEINEMANN AW, LOVELL LL 1987 Spinal cord injury rehabilitation outcome: the impact of age. Fournal of Chronic Diseases 41:173-177.

YARKONY GM, Roth EJ, HeINEMANN AW, WU Y, KATZ RT, Lovell L 1987 Benefits of rehabilitation for traumatic spinal cord injury: multivariate analysis in 711 patients. Archives of Neurology 44:9396.

Yarkony G, JAEGER R, WILliamson T, KRALJ A, QuinTERN J, Wu Y 1987 Standing by functional 
neuromuscular stimulation in the laboratory and at home: case reports of 18 paraplegic individuals. Proceedings of the 10th Annual Conference on Rehabilitation Technology 10:608-610.

YARKony GM, Roth EJ, HeINEMANN AW, Lovell L, WU Y 1988 Functional skills after spinal cord injury rehabilitation: three-year longitudinal followup. Archives of Physical Medicine and Rehabilitation 69:111-114.

Young JS, Burns PE, Bowen AM, McCutchen R, Thomas JP 1982 In: Spinal Cord Injury Statistics: Experience of the Regional Spinal Cord Injury Systems. Good Samaritan Medical Center, Phoenix, pp 1-10. 